\section{P001 DIFFERENTIAL EXPRESSION OF KEY METABOLIC GENES IN ANTIGEN-SPECIFIC B CELL SUBSETS IN RHEUMATOID ARTHRITIS AND SYSTEMIC LUPUS ERYTHEMATOSUS}

\footnotetext{
${ }^{1,2} \mathrm{~L}$ Abreu*, ${ }^{2} \mathrm{~F}$ Kucher, ${ }^{2} \mathrm{~V}$ Eckstein, ${ }^{2} \mathrm{H}-\mathrm{M}$ Lorenz, ${ }^{1} \mathrm{RA}$ Carvalho, ${ }^{2} \mathrm{M}$ Souto-Carneiro. ${ }^{1}$ Department of Life Sciences, University of Coimbra, Coimbra, Portugal; ${ }^{2}$ Department of Rheumatology, Universitätsklinikum Heidelberg, Heidelberg, Germany
}

\subsection{6/annrheumdis-2018-EWRR2019.1}

Career situation of first and presenting author Student for a master or a $\mathrm{PhD}$.

Introduction Antigen-specific B cells (Ag-Bs) differentiate into distinct subsets upon activation: plasmablasts (pBs, $\mathrm{CD} 19^{+} \mathrm{IgD}^{-} \mathrm{CD} 71^{+} \mathrm{CD} 38^{+} \mathrm{CD} 20^{-}$) and memory B cells (mBs, $\left.\mathrm{CD} 19^{+} \mathrm{IgD}^{-} \mathrm{CD} 71^{+} \mathrm{CD} 38^{\mathrm{dim}} \mathrm{CD} 20^{+}\right)$. The frequency of antigenspecific plasmablasts and memory $\mathrm{B}$ cells has been described to be altered in the blood of Rheumatoid Arthritis (RA) and Systemic Lupus Erythematosus (SLE) patients. Emerging evidence suggests that blood immune cells from RA and SLE patients exhibit different metabolic requirements and profiles when compared to healthy controls (HC). However, the metabolic configurations of these two Ag-Bs subsets in RA and SLE patients are yet to be described.

Objectives In the present study we aimed to characterize the gene expression of key metabolic enzymes in $\mathrm{mBs}$ and $\mathrm{pBs}$ from RA and SLE patients.

Methods Blood samples were obtained from 5 RA, 2 SLE and 3 HC donors. B cells were isolated and sorted into $\mathrm{pBs}$ and $\mathrm{mBs}$ populations. The gene expression of key metabolic enzymes was assessed by qPCR in both populations. The target genes analyzed were HIF1A, HK2, LDHA, MYC (glycolysis), CPT1A (fatty acid oxidation), SREBF1 (lipid synthesis) and PRKAA1 (mTOR inhibitor).

Results When comparing to $\mathrm{HC}$ there was a higher expression of: HIF1A, LDHA, MYC, PRKAA1 in RA mBs; HIF1A, HK2, LDHA, SREBF1 in RA pBs; HIF1A, HK2, LDHA, CPT1A, SREBF1 in SLE mBs; MYC and PRKAA1 in SLE pBs. When the gene expression was compared between RA and SLE patients, SLE $\mathrm{mBs}$ had a higher expression of $H K 2$, CPT1A and SREBF1 while SLE pBs exhibited higher levels of $M Y C$ and PRKAA1 expression. Thus, the observed results suggest that RA mBs, RA pBs and SLE mBs exhibit a glycolytic profile when compared to HC. SLE mBs also seem to have higher fatty acid oxidation and lipid synthesis levels when comparing to $\mathrm{HC}$ and $\mathrm{RA} \mathrm{mBs}$. The higher expression of SREBF1 in RA pBs could also indicate that lipid synthesis is upregulated in this subset. Interestingly, the results in SLE pBs suggest that all the analyzed metabolic pathways are downregulated when comparing to $\mathrm{HC}$ and RA pBs. Finally, since PRKAA1 is upregulated in RA $\mathrm{mBs}$ and SLE $\mathrm{pBs}$ one might hypothesize that inhibition of $\mathrm{mTOR}$ is greater in these subsets.

Conclusions The size of our sample is the principal limitation in this study and therefore we aim to increase it. Nevertheless, these preliminary results seem to indicate that antigenspecific B cells exhibit marked glycolytic profiles in RA and SLE patients. These results are expected in RA given the highly glycolytic requirements of $\mathrm{T}$ cells in this disease. Our study demonstrates that B cell metabolism should be characterized in depth in both RA and SLE.

Disclosure of Interest None declared.

\section{P002/026 DIFFERENCE BETWEEN PALINDROMIC RHEUMATISM AND EARLY RHEUMATOID ARTHRITIS AT THE LEVEL OF GENE EXPRESSION}

${ }^{1} \mathrm{~S}$ Ajaib*, ${ }^{2} \mathrm{~A}$ Chapman, ${ }^{1} \mathrm{~A}$ Burska, ${ }^{1} \mathrm{~K}$ Mankia, ${ }^{1} \mathrm{P}$ Emery, ${ }^{1} \mathrm{~F}$ Ponchel. ${ }^{1}$ LIRMM, University of Leeds, Leeds; ${ }^{2}$ University of Newcastle, Newcastle upon Tyne, UK

\subsection{6/annrheumdis-2018-EWRR2019.2}

Career situation of first and presenting author Student for a master or a $\mathrm{PhD}$.

Introduction Palindromic rheumatism (PR) is characterised by recurrent, episodic attacks of articular inflammation, which resolve completely without residual joint damage. Whether PR should be considered as a prodrome of rheumatoid arthritis (RA) or as a distinct syndrome remains unclear, over 70 years since first described. ${ }^{1}$ PR patients (non flare and during flare) were recruited in Leeds; RA and HC data was obtained from the RA-Map consortium. Differentially expressed genes (DEGs) were identified following a standard microarray analysis workflow. These findings reported the presence of distinct gene expression profiles within palindromic patients, compared to $\mathrm{HC}$ and those with drug naïve, early RA. ${ }^{2}$

Objectives To validate a 10 gene signature as a discriminant between PR and RA in order to better classify patients suspected of having $P R$ and to observe this signature as PR patients progress to early RA.

Methods PBMCs were taken from non-flare PR $(n=24)$; flare PR $(n=16) ;$ PR progressors to RA $(P R \rightarrow R A, n=10) ; \quad R A$ $(n=16)$; and patients suspected of having PR without an official diagnosis $(S p P R, n=31)$. All patients provided informed written consent. qPCR gene expression analysis was performed using 10 TaqMan assays and a house keeping gene.

Results Functional analysis of the DEGs revealed significantly enriched gene pathways ${ }^{2}$, from which a group of 10 DEGs with high expression fold differences between PR and RA were selected and grouped into three signatures: PR signature (high RPL10, low IL10, CCR6, UBC, and SHCBP1); RA signature (high ALPL, JUN and IFTIM2); Non-Flare vs Flare signature (high EIF3E, low HSPA9).

Comparing PR (non-flare and flare) with RA, 2 of the 5 genes (IL10, UBC) associated with PR were significantly different to RA $(p<0.05)$. For the RA signature, $2 / 3$ genes (JUN and IFITM2) were expressed significantly higher in RA $(\mathrm{p}<0.01)$. Comparing $\mathrm{PR} \rightarrow \mathrm{RA}$ with $\mathrm{RA}$, we observe higher levels of expression for 3/10 genes (ALPL, CCR6, SHCBP1, $\mathrm{p}=0.05$ ), and overall higher median values for 7 genes, not including IFITM2 and IL10 (suggesting nonRA).

Finally, comparing SpPR to $\mathrm{PR}$ and RA groups, we observed little differences in gene expression with PR; suggesting a correct diagnosis as no patients displayed a signature resembling that of RA. Nevertheless, one gene (JUN) was significantly higher in SpPR compared with PR, suggesting these patients may be in a more progressed disease state when presenting at the clinic.

Conclusions There are distinct differences (at the level of gene expression) between PR and RA, and those patients who progress to RA from $\mathrm{PR}$ display a molecular signature that remains closer to PR than RA. 